\title{
Anticancer drugs-induced toxicity in different age male CD-1 mice
}

Ana Reis-Mendes ${ }^{1}$, Margarida Duarte-Araújo ${ }^{2,3}$, José Alberto Duarte ${ }^{4}$, Salomé Gonçalves-Monteiro ${ }^{5}$, Fernando Remião ${ }^{1}$ Félix Carvalho ${ }^{1}$, Emília Sousa ${ }^{6,7}$, Maria Lourdes Bastos ${ }^{1}$, and Vera Marisa Costa ${ }^{1}$

1UCIBIO, REQUIMTE, Laboratory of Toxicology, Department of Biological Sciences, Faculty of Pharmacy, University of Porto, Porto, Portugal, 2LAQV/REQUIMTE, University of Porto, Portugal; ${ }^{3}$ Department of Immuno-Physiology and Pharmacology, Institute of Biomedical Sciences Abel Salazar, University of Porto, Portugal; ${ }^{4}$ CIAFEL, Faculty of Sports, University of Porto, Porto, Portugal; 5 LAQV/REQUIMTE, Laboratory of Pharmacology, Department of Drug Sciences, Faculty of Pharmacy, University of Porto, Porto, Portugal; ${ }^{6}$ Laboratory of Organic and Pharmaceutical Chemistry, Chemistry Department, Faculty of Pharmacy, University of Porto, Porto, Portugal; ${ }^{7}$ CIIMAR - Interdisciplinary Centre of Marine and Environmental Research, Porto, Portugal.

\section{INTRODUCTION}

Anticancer drugs like doxorubicin (DOX) and mitoxantrone (MTX) are used to treat cancer. Nevertheless, they have worrying long-term toxicity. MTX and DOX cause cardiotoxicity and several risk factors could aggravate it. The aim of this work was to study the toxicity of clinically relevant MTX and DOX doses in infant (4 weeks), adult (3 months), and old (18-20 months) male CD-1 mice.

\section{EXPERIMENTAL PROTOCOL}

ADMINISTRATION SCHEDULE:

- $1 \mathrm{mg} / \mathrm{kg}$ of saline $(\mathrm{NaCl}$ $0.9 \%)$, control group;

- MTX in saline (total cumulative dose of $6 \mathrm{mg} / \mathrm{kg}$ ).

- DOX in saline:

- first group: infants and adults (total cumulative dose of $18 \mathrm{mg} / \mathrm{kg}$ );

- second group: adults and old (total cumulative dose of 9 $\mathrm{mg} / \mathrm{kg}$ ).

Biweekly intraperitoneal administrations, for 3 weeks were given to all animals.

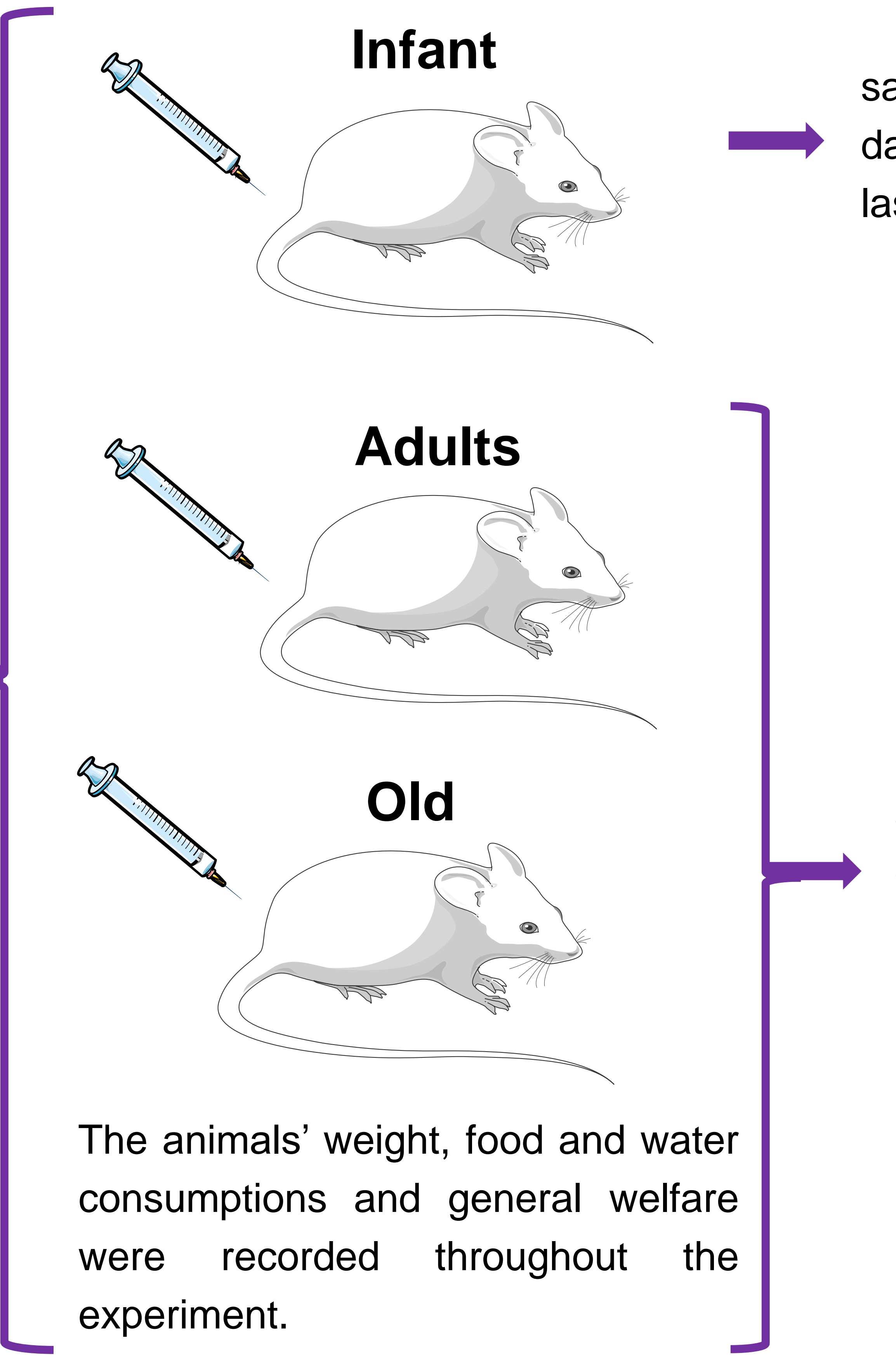

sacrificed 17 days after the last injection

The organs were removed and weighted and blood plasma was collected.

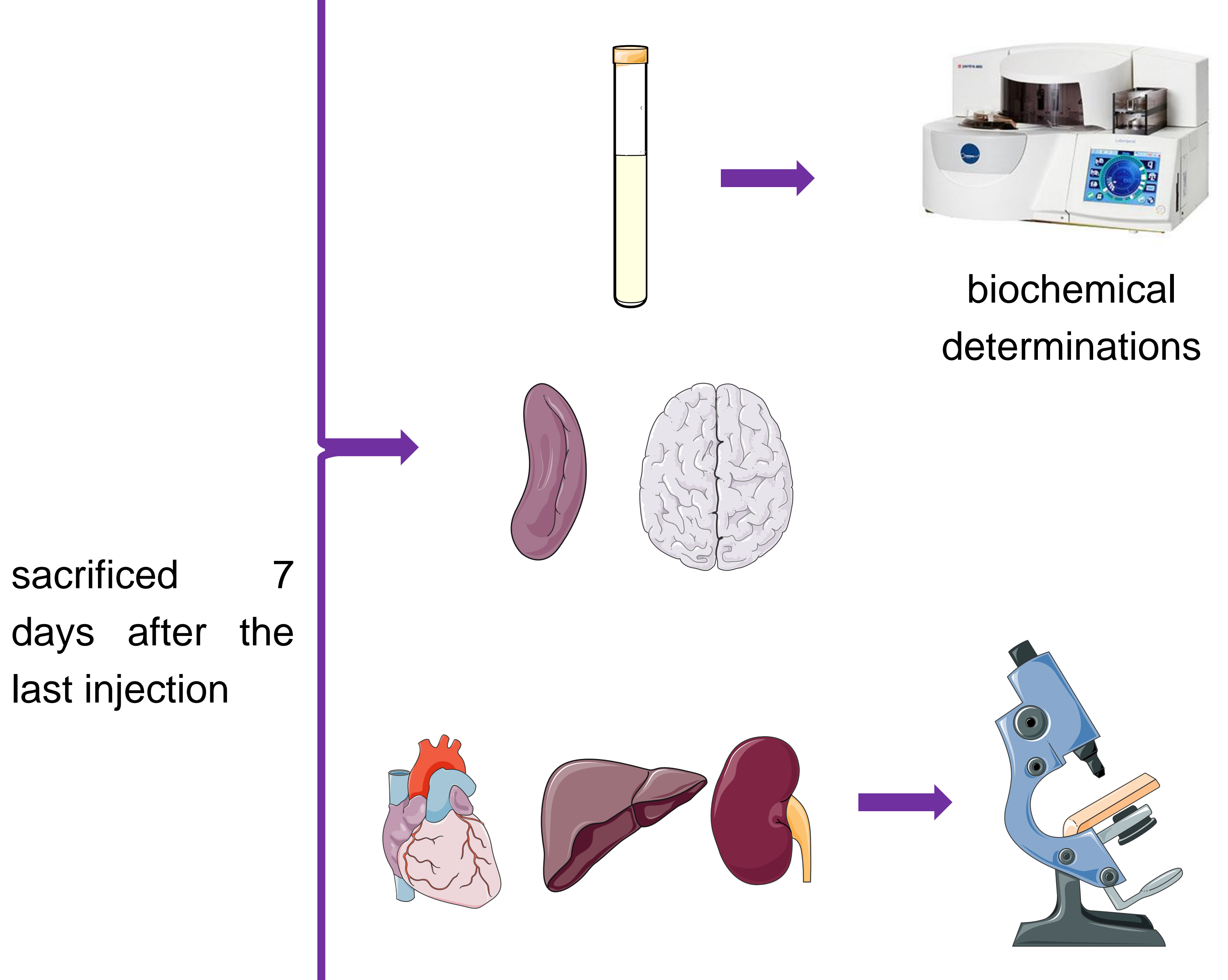

light microscopy

\section{RESULTS}

Regarding the results, significant body weight loss was observed in the adult population treated with MTX and DOX $18 \mathrm{mg} / \mathrm{kg}$, and in DOX-treated infants' animals, when compared to the respective controls. In addition, food and water consumptions decreased in the infant and adult populations treated with MTX and DOX $18 \mathrm{mg} / \mathrm{kg}$. Regarding organ weight/ brain weight ratios at sacrifice, the ratio of all the organs were significantly decreased in the MTX and DOX-treated adults, except in adult DOX $9 \mathrm{mg} / \mathrm{kg}$. Alanine aminotransferase (ALT) plasma levels were significantly increased in the MTX and DOX-treated infants. Moreover, in DOX-treated adults $(18 \mathrm{mg} / \mathrm{kg}$ ), aspartate aminotransferase (AST)/ALT ratio and total creatine-kinase (CK) plasma levels increased, when compared to controls. Regarding histology, all the MTX and DOX-treated populations presented lesions in heart, kidneys, and liver as did the old control animals. Histological staining using Sirius Red identified a significant increase in interstitial collagen deposition in the myocardium, liver, and kidneys in all drug-treated adult mice.

\section{DISCUSSION AND CONCLUSIONS}

Therefore, we can conclude that although cumulative dose is considered a main factor when studying anticancer drugs toxicity, the age of administration is also determinant in the toxicity observed. In our study, adult mice seemed to be more prone to MTX and DOX-induced toxicity; still, further data needs to be gathered to determine the underlying factors for that susceptibility.

\section{ACKNOWLEDGEMENTS}

ARM and VMC acknowledge Fundação da Ciência e Tecnologia (FCT) for their grants (SFRH/BD/129359/2017 and SFRH/BPD/110001/2015, respectively). This work is funded by national funds through FCT - Fundação para a Ciência e a Tecnologia, I.P., under the Norma Transitória - DL57/2016/CP1334/CT0006. This work was supported by FEDER funds through the Operational Programme for Competitiveness Factors - COMPETE and by national funds by the Fundação para a Ciência e Tecnologia (FCT) within the project "PTDC/DTP-FTO/1489/2014 - POCI01-0145-FEDER-016537". 